\title{
Classic Craft: Fuzhou Bodiless Lacquer Ware
}

\author{
Xin Zheng ${ }^{1, *}$
}

\author{
${ }^{1}$ Minjiang University, Fuzhou, Fujian, China \\ *Corresponding author. Email: zhengxin1968@126.com
}

\begin{abstract}
Fuzhou lacquer art occupies important position in the field in China. The most representative one is Fuzhou bodiless lacquer ware, which is one of the three treasures of Fuzhou arts and crafts with delicate shape, solid quality, rich color, and long lifetime. The time-honored craft of bodiless lacquer ware is similar to that of fabric core lacquer of Han Dynasty. Based on the traditional craft of "fabric core", Shen Shaoan has come up with new ideas to elevate the craft to a new height. The author summarizes the development of Fuzhou bodiless lacquer ware, and clearly expounds its technological characteristics.
\end{abstract}

\section{Keywords: bodiless, lacquering, craft, foundation}

\section{INTRODUCTION}

With long history and superb craftsmanship, Fuzhou arts and crafts have long enjoyed the reputation of "three mountains of fine arts, unique in the world". It's no exaggeration to say that Fuzhou craftsmen have played an important role in the development and innovation of almost all the iconic crafts in the history of Chinese civilization. Bodiless lacquer ware, Shoushan stone sculpture and cork picture are the most distinctive one with brilliant achievements.

Fuzhou is an important industrial town of modern lacquer art in China with profound lacquer culture. Song Dynasty witnessed the uprising of Fuzhou lacquer art, making Fuzhou an important area of China's folk carved lacquer ware. During the Song, Yuan, Ming and Qing dynasties, Fuzhou lacquer art has been exported overseas as major export commodity, which accordingly prompts the dissemination of lacquer culture. In the mid-Qing Dynasty, Fuzhou's brand of lacquer art - "bodiless lacquer ware" rose to fame, reinforcing Fuzhou's historical status of "the center of Renaissance" of Chinese lacquer art.

\section{THE TILES BODY OF BODILESS LACQUER} WARE

The representative of Fuzhou lacquer ware is "bodiless lacquer ware", which is named after the tiles bodies of lacquer ware. To understand lacquer well, one must understand the manufacturing technique for tiles bodies. The reason why the emphasis on the concept of "body" is because the paint cannot be formed alone, must adhere to a medium, body. The most familiar body is made of wood, employed by most lacquer ware. There exists also leather, bamboo, metal, ceramic

bodies and so on. The pottery lacquer ware is especially heavy because of the weight of the body inside.

Then what is "bodiless"? The professional expression is "take off the mud body and remain cloth body". The simple operation process is as follows: first, clay modeling (clay body); second, pasting linen (cloth body); third, scraping and painting (repairing cloth body); fourth, removing mud body (remaining cloth body). There are many ways to remove the mud body, the most traditional of which is to soak it in water, the mud body dissolved from the shell, leaving cloth body made of paint gray and linen cloth. Therefore, "bodiless" refers to the conversion of two media through detachment (desilting) method, to transform the mud body into cloth one. This is a bodiless techniques with highest technical content in Chinese traditional lacquer.

The greatest advantage of "bodiless" lies in its lightness. It is light and easy to move. It has been used to create giant Buddha statues since Southern and Northern Dynasties. According to historical records, the largest "bodiless lacquer statues" in Northern Wei was as high as several zhang, a unit of length (31/3 meters), which could hold dozens of flag bearers. It became more popular in Tang Dynasty, with large-scale monasteries boasting "bodiless lacquer statues". During Buddhist celebrations, believers welcome the Buddha out of temple and carry him around the streets. The stone-made one is too heavy to lift, let alone parade in the streets. However, "bodiless lacquer statues" looks heavy and powerful but is light and easy to move, especially suitable for such occasions. Then why is it now so rare? Given its lightness, most of China's "bodiless lacquer statues" was shipped overseas after the Opium War. So far, the most well-preserved "bodiless lacquer statues" of the Tang Dynasty is the 
main Buddha of Toshodai Temple in Nara, Japan - the Statue of Vairochana Buddha created by Tan Jing, a monk of ChaoGong Temple in Quanzhou, Fujian, who traveled to Japan with Jianzhen, which indicates that the "bodiless" technology then and there has reached maturity.

The craft of "bodiless" was first used in lacquer of the Warring States Period. What people then called it is not clear. In the Western Han Dynasty, it was called "fabric core", which is different from other dynasties. Different in name, "fabric core" and "bodiless" share technique. It was not until after the Qing Dynasty that the latter appeared. Emperor Qianlong wrote a poem praising the antique lacquer ware made by Suzhou craftsmen in the Qing dynasty, in which the word "bodiless" was first recorded. Based on the traditional craft of "fabric core", Shen Shaoan, a lacquer artist from Fuzhou in the Qing Dynasty, has made continuous attempts and bold innovations to push the traditional craft of lacquer to a new height. As a result, he is respected by later generations as the ancestor of Fuzhou "bodiless lacquer", and the lacquer made him as "bodiless lacquer".

\section{THE LACQUER OF BODILESS LACQUER}

The professional term for lacquer is "Xiu Qi Zhi Qi (utensils made of lacquer)". The interpretation of "Xiu" is "paint". So the common definition of lacquer ware is "painted lacquer ware". The predecessor of lacquer ware was wood ware, which was also called "Mu Qi Xiu Zhe" in the Western Han Dynasty. Painting on wood is the earliest form of ancient Chinese lacquer ware, which is also the most basic structure of lacquer ware in China and other countries.

Lacquer in lacquer ware mainly refers to the natural raw lacquer, which is the lacquer cut on lacquer tree, commonly known as big lacquer. "桼 (Qi)" in the ancient oracle means "漆 (lacquer)". Although the radical is omitted, the structural pattern of "木 (wood) on the top, 人 (people) in the middle and 水 (water) under" has remained. The "木" stands for lacquer tree. Adult lacquer tree can be up to 30 meters tall. Immature trees can't be cut, so it makes sense to stand on top. "人" stands for lacquer farmer. This is the best part of the character. If you pick the paint at the lower end, it will hurt the tree. If you pick the paint at the upper end, the lacquer will be of poor quality. Only lacquer farmers will climb to the middle of the lacquer tree. "水" stands for lacquer. Lacquer containers are only suitable for placing under trees. It makes sense to put 水 under. The word "漆" accurately and vividly depicts the working scene of picking and cutting lacquer, indicating the relationship among people, lacquer tree and lacquer.
Lacquer itself is a natural coating. Thanks to its moisture resistance and corrosion-proof, it is employed to avoid the decay and deformation of wooden objects, thus extending their service life. On this basis, the lacquer can also be used to decorate, beautify utensils.

Lacquer is the first procedure of lacquer decoration. The rest of decoration is based on the paint. What is its original color? The natural lacquer is tan after oxidation in contact with air. It turns black after a few more strokes. Hence the word "pitch-black". Black became the main color. Therefore, if there is no special mention of color when talking about ancient lacquer ware, it is black.

When lacquer is added to vermilion (mercury sulfide), red paint came into being. Since ancient times, red paint and black paint are the best partners. Most early lacquer ware in China was black and red. Han Fei stated in Shi Guo Pian that "Yu made sacrificial vessel with black paint on the outside and red paint on the inside".

Light paint is a unique innovation of Shen Shaoan family with special color effect. Western scholars praised that: "If gold and silver dust with best quality are mixed in paint, the finish enjoys pleasant gloss and strong artistic appeal." What is light paint? As a decoration material: it is mixed with gold and silver clay (finely grinded with gold and silver foil). Due to the gold and silver material, it's very expensive, it glows beautifully with metal. As for genre, it is painted with thumb rackets (the traditional method of heavily painting colors with brushes is a waste of gold and silver), so the paint is extremely thin and the surface is extremely delicate. The recipe and operation of the light paint have long been regarded as a unique skill by the Shen family. The high-end products are almost always decorated with light lacquer ware, such as the Bamboo Root Bottle, Lotus Leaf Bottle and Basket Fairy (preserved in the Fujian Museum), all of which are known as the three treasures of bodiless lacquer ware. Thanks to light lacquer, the color of traditional lacquer also covers a series of gorgeous and elegant bright color besides commonly-seen red, gold, and black. In this way, a whole set of unique representation system has been formed from painting technique to decoration technique.

\section{THE HISTORY OF BODILESS LACQUER} WARE

Fuzhou is home to bodiless lacquer ware, which has profound lacquer culture. The history of Fuzhou lacquer art can be traced back to the Warring States Period more than two thousand years ago. Archaeological data show that "lacquer remains and skin" were found in the two excavations of Chu Tomb in Minhou Village, Fuzhou. 
In the Five Dynasties, Fuzhou established a "Hundred Handicraft Academy", which brought together skilled artisans (including painters) from all walks of life and specialized in producing all kinds of high-grade handcrafts for the ruling class. Historical records show that in the first year of Tongguang (923), there was a painting box made in Fuzhou, which was supposed to be made by the academy due to its mass production.

The Song Dynasty laid the foundation for the revival of Fuzhou lacquer art. With the support of the government and the development of overseas trade, Fuzhou has become an important producing area for lacquer production in China. Literature shows that Fuzhou mother-of-pearl (lacquer ware inlaid with shells) has exquisite workmanship and diversified forms. The unearthed objects show that Fuzhou carved lacquer ware (lacquer ware carved with concave and convex patterns) is numerous in number, skilled and distinct.

In the Ming and early Qing dynasties, "Wo lacquer" (Japanese lacquer) was introduced into Fuzhou, which was called "foreign lacquer" (Oriental lacquer). In Ming Dynasty, Fuzhou lacquer artist Yang Xun was excel at making "Wo lacquer" and made bold innovations to form his own unique style, known as "Yang Wo". During the Reign of Emperor Yongzheng and Qianlong, Fuzhou imitated foreign lacquer, which was recorded in the Qing archives. List of Tributes to Imperial Palace even details the specific batch and quantity. Chinese carved lacquer was introduced to Japan in the early Ming Dynasty and was favored by the upper nobility, which led to the imitation of Japanese craftsmen. The "Yuntai carving", with the style of Fujian carving, was popular for a time. Historical records indicate that Fujian carving, represented by Wei Ruanlan and Wang Mengming, enjoyed good reputation in Fuzho in the late Ming and early Qing dynasties.

During the Reign of Qianlong in the Qing Dynasty, the appearance of Shen Shaoan family changed the quality of Fuzhou lacquer art. Shen Shaoan and his descendants have restored the lost "bodiless lacquer" and developed the technique of patting light lacquer. They have also borrowed the advantages of Japanese "Wo lacquer" and created unique "bodiless lacquer", which is one of the most brilliant pieces of Fuzhou lacquer art. Fuzhou is also one of the cradles of higher education of Chinese lacquer craft. In 1907 Fuzhou Arts and Crafts Institute was established, lacquer department (east lacquer and Chinese lacquer) was set up with Japanese Harata and Lin Hongzeng, royal worker of Qing Dynasty, as teachers. A large number of lacquer artists were trained, including the pioneer of modern Chinese lacquer art, Li Zhiqing. At the end of Qing Dynasty and the beginning of The Republic of
China, Shen's lacquer ware shops were scattered in Fuzhou, covering Shen Zhenghao's "Zheng Ji" (also called "Hao Ji"), Shen Zheng Xun's "Xun Ji", Shen Youlan's "Lan Ji", Shen Zhengkai's "Kai Ji", Shen Zhengyu's "Yu Ji" and Shen Zhengxi's "Xi Ji". Shen Zhenghao, Shen Zhengxun and Shen Youlan (Zhengyi) attended several international exhibitions and won various honors, which made Fuzhou's "bodiless lacquer" well known.

\section{CONCLUSION}

Fuzhou's bodiless lacquer ware is the symbol of Fuzhou regional culture, which highlights the profound cultural deposits of Rong City. Thanks to the unremitting efforts of hundreds of skilled artisans, Fuzhou bodiless lacquer ware has formed its own unique craft, whose unique skills and exquisite workmanship are marveled by experts in the field of Chinese lacquer art. Considering its high artistic research value, it is the due responsibility to inherit and carry forward such classic technique.

\section{References}

[1] He Mianshan. Fujian Religious Culture [M]. Tianjin: Tianjin Academy of Social Sciences, 2004:37. (in Chinese)

[2] He Haoliang, Tao Shizhi. Lacquer Carving Crafts [M]. Fuzhou: Fujian Fine Arts Publishing House, 1989:10. (in Chinese)

[3] Fujian Museum, Shaowu Museum. Excavation of Huanghuan Tomb of the Song Dynasty in Shaowu [J]. Fujian Wenbo, 2004(2): 7. (in Chinese)

[4] Lin Gongxiang. On the Infinite Possibilities of Lacquer [J]. Art Life, 2005(6):10. (in Chinese)

[5] Qiao Shiguang. Newborn Chinese Lacquer Painting [C] Editorial Committee of Complete Works of Chinese Modern Art. Complete Works of Chinese Modern Art: Lacquer Painting. Beijing: People's Fine Arts Publishing House. 1998:13. (in Chinese)

[6] The Dictionary Editing Office of the Institute of Languages, Chinese Academy of Social Sciences. Modern Chinese Dictionary (Revised Edition). Beijing: The Commercial Press, 1978:1 417. (in Chinese)

[7] (Ming) Huang Cheng, Yang Ming. Records of Lacquering [M] Wang Shixiang. Interpretation of Records of Lacquering. Beijing: Cultural Relics Publishing House, 1983. (in Chinese) 